\title{
Florid Cemento-Osseous Dysplasia: A Case Report
}

\author{
Kaan GÜNDÜZ1 \\ Hakan AVSEVER ${ }^{2}$ \\ Ümit KARAÇAYLI ${ }^{3}$ \\ Buğra ŞENEL ${ }^{2}$ \\ Bülent PIŞKINN ${ }^{4}$
}

\author{
${ }^{1}$ Department of Oral Diagnosis and Radiology, Ondokuz Mayls University, Samsun,Turkey \\ ${ }^{2}$ Department of Oral Diagnosis and Radiology, Gulhane Military School of Medicine, Ankara, Turkey \\ ${ }^{3}$ Department of Oral and Maxillofacial Surgery, Gulhane Military School of Medicine, Ankara, Turkey \\ ${ }^{4}$ Department of Prosthodontics, Van Military Hospital, Van, Turkey
}

\begin{abstract}
Florid cemento-osseous dysplasia (FCOD) has been described as a condition that characteristically affects the jaws of middle-aged black women. It usually exhibits as multiple radiopaque cemetum-like masses distributed throughout the jaws. Radiographically, FCOD appears as dense, lobulated masses, often symmetrically located in various regions of the jaws. Computed tomography, because of its ability to give axial, sagittal, and frontal views, is useful in the evaluation of these lesions. This paper presents the case of a patient who was diagnosed with FCOD on the basis of clinical and radiographic findings.
\end{abstract}

Key Words: florid cemento-osseous dysplasia, gigantiform cementoma, sclerosing osteitis, multiple enostoses, diffuse chronic osteomyelitis.

\section{INTRODUCTION}

The term florid cemento-osseous dysplasia (FCOD) refers to a group of fibro-osseous (cemental) exuberant lesions with multi-quadrant involvement (1). FCOD is a very rare condition presenting in the jaws. These lesions are most commonly seen in middle-aged black women, although it also may occur in Caucasians and Asians $(2,3)$, and have been entitled as sclerosing osteitis, multiple enostoses, diffuse chronic osteomyelitis and gigantiform cementoma $(3,4)$. The cause of FCOD is still unknown, and there is no satisfactory explanation for the reported gender and racial predilection (2-5).

FCOD lesions have a striking tendency towards bilateral, often quite symmetrical, location, and it is not unusual to find extensive lesions in all 4 posterior (molarpremolar region) quadrants of the jaws (5). Clinically, these lesions are often asymptomatic and may present as incidental radiological findings. Symptoms such as dull pain or drainaige are almost always associated with exposure of the sclerotic calcified masses in the oral cav- ity (3-5). This may emerge as the result of progressive alveolar atrophy under a denture or after extraction of teeth in the affected area involved (5).

Radiographically, FCOD appear as dense, lobulated masses, often symmetrically located in various regions of the jaws $(6,7)$. Computed tomography $(\mathrm{CT})$, because of its ability to give axial, sagittal, and frontal views, is useful in the evaluation of these lesions $(8,9)$.

This paper presents the case of a patient who was diagnosed with FCOD on the basis of clinical and radiographic findings.

\section{CASE REPORT}

A 43-year-old Caucasian female patient was admitted to our Department of Oral Diagnosis and Radiology with a complaint of acute pain in the left molar region of the maxilla. The patient was systemically healthy and her physical examination showed no significant abnormality. Intraoral examination revealed full crown ceramic prosthetic restorations on both maxilla 
and mandible and a partially edentulous area in the left mandible. Teeth were non-tender to percussion except for the left maxillary second molar. The left maxillary second molar exhibited significant degree of mobility. On palpation, there was bilateral buccal bone expansion noted on posterior mandible. The overlying gingiva and mucosa in mandible were normal without any clinical signs of inflamation. Orthopantomograph showed diffuse, lobular, irregularly shaped radiopacities or cottonwool appearance throughout the alveolar process of the both quadrants of the maxilla and mandible (Fig. 1). Cyst-like radiolucent areas were observed around some radiopaque lesions. There was no root resorption or fusion of the lesions to the involved teeth. Periapical radiograph

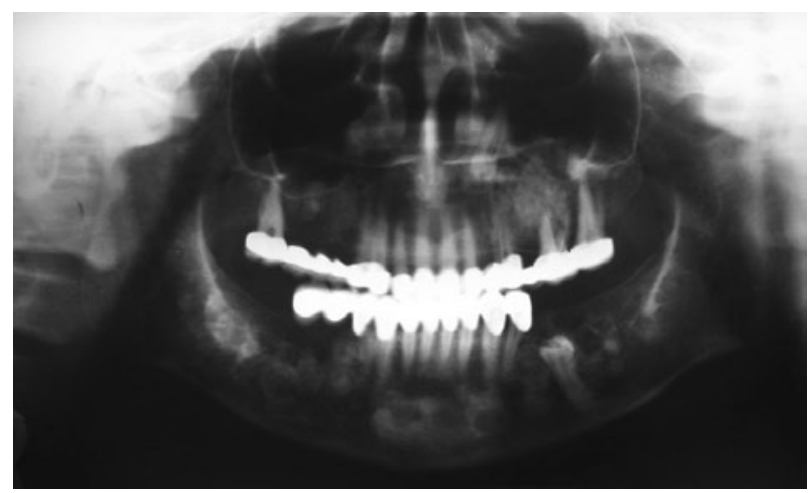

Figure 1. Panoramic radiograph showing diffuse, lobular, irregularly shaped radiopacities or cotton-wool appearance throughout the alveolar process of the both quadrants of the maxilla and mandible.

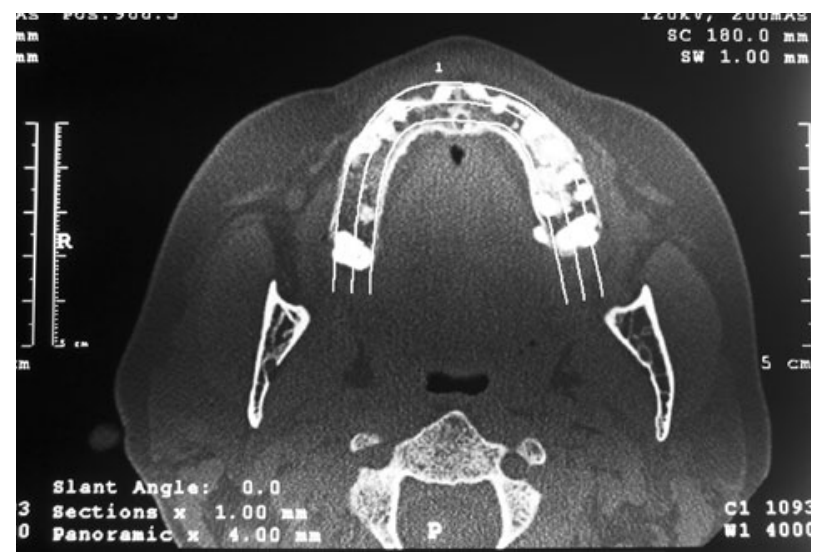

Figure 3. Axial CT scan showing high-density masses and buccolingual bone expansion in premolar-molar region of left maxilla. showed presence of periodontal bone defect in the left maxillary second molar. Additionally, the left maxillary canine and the right mandibular premolar were impacted.

When these findings were compared with a panoramic radiograph taken one year earlier, it appeared that no radiographic modifications had occurred. The lesion was supposed to be FCOD, however in the absence of a more specific diagnosis a decision was made to perform additional imaging. Axial CT scans showed buccolingual bone expansion in posterior maxilla and mandible (Figs. 2 and 3). The relationship between the mandibular canal and the pathologic masses was visualized. Panoramic-like reconstructions showed bilateral extensions in to the floor of the antrum and at the level of the root apices were presented on both maxilla and mandible (Fig. 4).

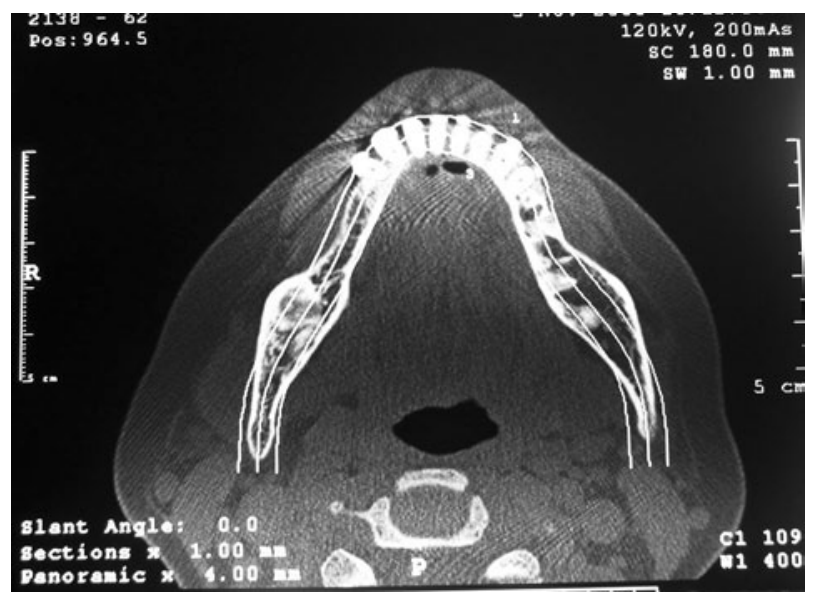

Figure 2. Axial CT scan showing high-density masses and bilateral buccolingual bone expansion in posterior mandible.

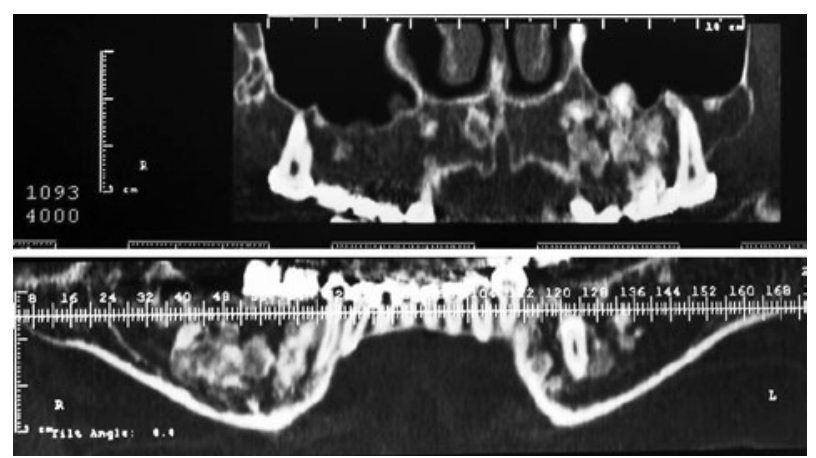

Figure 4. Panoramic-like reconstructions showing root apices, mandibular nerve, their relationship to radiopaque masses and bilateral extensions in to the floor of the antrum. 
Complete blood count and other laboratory data were within normal limits. Biopsy was not done as the case was diagnosed as FCOD on the basis of the characteristic features seen on the radiographs. Because the periodontal defect and the mobility, a decision was made to extract the left maxillary second molar. The case has been followed up over the last 11 months. Patient is asymptomatic.

\section{DISCUSSION}

Exuberant fibro-osseous lesions occurring in multi-quadrants of the jaws were designated as gigantiform cementomas or familial multiple cementomas in the first edition of the World Health Organization's Histological Typing of Odontogenic Tumours, Jaw cysts and Allied Lesions (10). Melrose et al. (4) introduced the term florid osseous dysplasia. The authors, however, prefer the term FCOD proposed by Waldron (3) because the dense, sclerotic masses closely resemble cementum. This term was also advocated by Kramer et al. (11) in the most recent histological classification of odontogenic tumors.

In our school, we have diagnosed cases of FCOD based on clinical findings, localization of lesions, and on the patient's age, gender, and ethnicity, as well as radiological features. Since our patients have remained asymptomatic, the lesions have not been subjected to biopsy procedures. And as reassuring a biopsy might be in this condition, it may precipitate infection that is difficult to control without extensive surgical intervention. Several authors have observed that patients presented with poor socket healing and even sequestrum formation following extraction of teeth. It is recommended that every effort should be made to avoid extraction or even elective surgical procedures. After the initial acute lesion in the maxillary molar region resolved, the clinical situation was unremarkable. Clinical examination was normal, with no spontaneous pain or dental sensitivity. All teeth were vital.

FCOD is a reactive, non-neoplastic process confined to tooth-bearing areas of the jaws that is seen most frequently in middle-aged and older women of African descent $(3,10)$. Melrose et al. (4) reported a study of 34 cases of such lesions, of which 32 were black women (in a predominantly Caucasian population) with a mean age of 42 years. In the Oriental population, Loh and Yeo (12) reported 9 cases diagnosed over a 34-year period, of whom 8 were middle-aged Chinese women and the other was an Indian woman. The definite female gender predilection of the condition cannot be explained. In the present case, the patient was a 43-year-old Caucasian female.

When patients are asymptomatic, conventional radiographs that exhibit multiquadrant diffuse radiopaque masses in the alveolar parts play an important role in the diagnosis. The lesions are typically found in the toothbearing areas of the jaws. The radiographic appearance, though not pathognomonic, is quite characteristic and very helpful in establishing the diagnosis (13). The use of CT has been previously reported $(5,8)$. Axial CT images clearly show the location and extent of the lesion, especially in the maxilla. The expansion of the cortical bones is clearly evaluated on $\mathrm{CT}$, even if it is slight. CT can be used to differentiate FCOD from lesions that exhibit a similar sclerotic appearance on conventional radiographs. In enostosis or exostosis, the high-density masses are more clearly observed on axial CT images than on occlusal radiographs, and they are found to be continuous with cortical plates (8). Odontogenic tumors, especially cemento-ossifying fibroma, usually exhibit more buccolingual expansion than does FCOD (14). Paget's disease of the bone may have a cotton-wool appearance (15). On the other hand, this condition affects the bone of the entire mandible and exhibits loss of lamina dura, whereas FCOD is localized above the mandibular canal and 2 thirds of its cervical are normal (16). Paget's disease is also characterized by deformities of multiple bones and produces biochemical serum changes, such as elevated alkaline phosphate levels (12). No biochemical alterations and other bone involvement were found in this case presented. Differential diagnosis of FCOD should also include sclerosing osteomyelitis, which can be a complication of the disease $(5,17,18)$.

Once the diagnosis has been established in an asymptomatic patient, under normal circumstances, there is no need to exist further treatment. The patient should be regularly follow-up and recall examinations with prophylaxis and reinforcement of good home hygiene care to control periodontal disease and prevent tooth loss $(5,13)$. In the absence of clinical signs, reevaluation with panoramic or CT imaging every 2 or 3 years is adequate (5). Management of the symptomatic patient is more difficult because chronic inflammation and infection develop within densely mineralized tissue. Generally antibiotics are not effective in FCOD so 
their tissue diffusion is poor. Indeed biopsy increases the risk of infection or may cause jaw fractures and it is not normally justified to surgically remove these lesions as this often requires extensive surgery $(2,5-8,13)$.

Normally, a diagnosis of FCOD in the jaws is made by clinical findings, radiographic features and histology. However, this is a condition in which the diagnosis relies on radiological and clinical findings alone. As reassuring as a biopsy might be, in this situation, it may precipitate infection that is difficult to control without extensive surgical intervention. The patient of the present case has been followed up over the last 11 months and FCOD has remained asymptomatic.

\section{RESUMO}

Adisplasia cemento-óssea florida (DCOF) tem sido descrita como uma condição que afeta tipicamente os maxilares de mulheres negras de meia idade, geralmente exibindo massas radiopacas semelhantes ao cemento, distribuídas nos ossos maxilares. Radiograficamente, a DCOF apresenta-se como densas masas lobuladas, frequentemente distribuídas simetricamente em diversas regiões dos maxilares. A tomografia computadorizada, que oferece vistas axial, sagital e frontal, é útil na avaliação dessas lesões. Este artigo apresenta o caso de uma paciente em que a DCOF foi diagnoasticada com base nos achados clínicos e radiográficos.

\section{REFERENCES}

1. Siew-Tin O, Chong-Huart S. Florid cemento-osseous dysplasia in a young Chinese man. Case report. Aus Dent J 1997;42:404-408.

2. Miyake M, Nagahata S. Florid cemento-osseous dysplasia. Report of a case. Int J Oral Maxillofac Surg 1999;28:56-57.

3. Waldron CA. Fibro-osseous lesions of the jaws. J Oral Maxillofac Surg 1985;43:249-262.

4. Melrose RJ, Abrams AM, Mills BG. Florid osseous dysplasia: a clinical-pathological study of thirty-four cases. Oral Surg 1976;41:62-82.
5. Beylouni I, Farge P, Mazoyer JF, Coudert JL. Florid cementoosseous dysplasia. Oral Surg Oral Med Oral Pathol Oral Radiol Endod 1998;85:707-711.

6. Damm DD, Fantasia JE. Multifocal mixed radiolucencies. Florid cemento-osseous dysplasia. Gen Dent 2001;49:461.

7. Goncalves M, Ronaldo P, Alves FA, Blugao CB, Gonalves A. Clinical, radiographic, biochemical and histological findings of florid cemento-osseous dysplasia and report of a case. Braz Dent J 2005;16:247-250.

8. Ariji Y, Ariji E, Higuchi Y, Kubo S, Nakayama E, Kanda S. Florid cento-osseous dysplasia. Oral Surg Oral Med Oral Pathol Oral Radiol Endod 1994;78:391-396.

9. Abdelsayed R, Eversole L, Singh B, Scarbrough F, Augusta G. Gigantiform cementoma: clinicopathologic presentation of 3 cases. Oral Surg Oral Med Oral Pathol Oral Radiol Endod 2001;91:438444.

10. Pindborg J, Kramer I, Torloni H. Histological typing of odontogenic tumors, jaw cysts and allied lesions. International histological classification of tumors. Geneva: World Health Organization 1971:32-34.

11. Kramer I, Pindborg J, Shear M. Histological typing of odontogenic tumours. $2^{\text {nd }}$ ed. London: Springer-Verlag 1992;29-30.

12. Loh F, Yeo J. Florid osseous dysplasia in Orientals. Oral Surg Oral Med Oral Pathol 1989;68:748-753.

13. Summerlin D, Tomich C. Focal cemento-osseous dysplasia: a clinicopathologic study of 221 cases. Oral Surg Oral Med Oral Pathol 1994;78:611-620.

14. DelBalso A, Werning J. The role of computed tomography in the evaluation of cemento-osseous lesions. Oral Surg Oral Med Oral Pathol 1986;62:354-357.

15. Minhas G, Hodge T, Gill DS. Orthodontic treatment and cementoosseous dysplasia: a case report. J Orthod 2008;35:90-95.

16. Langlais R, Langlan O, Nortje C. Diagnostic imaging of the jaws. 1st ed. Malvern: Williams \& Wilkins;1995.

17. Cavalcante AS, Sgarbi FC, Agapito L da C, Roveroni LH, Brandao AA, Cabral LA. Florid cemento-osseous dysplasia: a report of three cases. Gen Dent 2008;56:186-190.

18. Islam MN, Cohen DM, Kanter KG, Stewart CM, Katz J, Bhattacharyya I. Florid cemento-osseous dysplasia mimicking multiple periapical pathology-an endodontic dilemma. Gen Dent 2008;56:559-562. 